\title{
Allicin inhibits the invasion of lung adenocarcinoma cells by altering tissue inhibitor of metalloproteinase/matrix metalloproteinase balance via reducing the activity of phosphoinositide 3-kinase/AKT signaling
}

\author{
LING HUANG $^{1}$, YUANHONG SONG ${ }^{2}$, JIANPING LIAN ${ }^{3}$ and ZHIWEI WANG ${ }^{1}$ \\ ${ }^{1}$ Department of Cardiovascular Surgery, Renmin Hospital, Wuhan University, Wuhan, Hubei 430060; Departments of \\ ${ }^{2}$ Pathology and ${ }^{3}$ Oncology, The Affiliated Hospital of Jinggangshan University, Jian, Jiangxi 343000, P.R. China
}

Received February 16, 2016; Accepted February 7, 2017

DOI: 10.3892/ol.2017.6129

\begin{abstract}
Allicin, the main active principle associated with Allium sativum chemistry, has various antitumor activities. However, to the best of our knowledge, there is no available information to address the anti-invasive effect and associated mechanism in lung adenocarcinoma. In the present study, cell viability assay, cell adhesion assay, western blot analysis, Transwell migration and invasion assays and reverse transcription-quantitative polymerase chain reaction were performed. Allicin was identified to inhibit the adhesion, invasion and migration of lung adenocarcinoma cells in a dose-dependent manner, accompanied by decreasing mRNA and protein levels of matrix metalloproteinase (MMP)-2 and MMP-9. Conversely, the mRNA and protein levels of tissue inhibitor of metalloproteinase (TIMP)-1 and TIMP-2 were increased in a dose-dependent manner. Furthermore, it was revealed that allicin treatment significantly suppressed the phosphorylation of AKT $(\mathrm{P}<0.05)$, but not the total protein expression of AKT. Combined treatment with LY294002, an inhibitor of phosphoinositide 3-kinase (PI3K)/AKT signaling, and allicin led to the synergistic reduction of MMP-2 and MMP-9 expression, followed by an increase in TIMP-1 and TIMP-2 expression. The invasive capabilities of lung adenocarcinoma cells were also suppressed. However, insulin-like growth factor-1 (an activator of PI3K/AKT signaling) reversed the effects of allicin on cell invasion and expression of MMP-2, MMP-9, TIMP-1 and TIMP-2. The present study concluded that allicin may inhibit
\end{abstract}

Correspondence to: Professor Zhiwei Wang, Department of Cardiovascular Surgery, Renmin Hospital, Wuhan University, 99 Zhang Zhidong Road, Wuhan, Hubei 430060, P.R. China

E-mail: zhiweiwang6666@126.com

Key words: allicin, phosphoinositide 3-kinase, AKT, matrix metalloproteinase-2, matrix metalloproteinase-9, tissue inhibitor of metalloproteinase-1, tissue inhibitor of metalloproteinase-2, lung adenocarcinoma, invasion invasion of lung adenocarcinoma cells by altering TIMP/MMP balance, via reducing the activity of the PI3K/AKT signaling pathway. This indicated that allicin may be recognized as an anti-invasive agent for lung adenocarcinoma treatment.

\section{Introduction}

Lung cancer is the predominant reason of cancer-associated mortality in developed and developing countries. Lung adenocarcinoma is the most common histological type of lung cancer, and accounts for $\sim 50 \%$ of all lung cancers (1). Although the management and treatment of surgery, radiotherapy and chemotherapy have improved, the therapeutic efficacy is poor. One of the major reasons is the lack of adequate treatment against lung adenocarcinoma invasion $(2,3)$. Therefore, the development of novel adjuvant therapeutic strategies specifically targeting the progression of invasion is of critical importance for improving the prognosis of patients with lung adenocarcinoma.

Invasion occurs through a complex pathophysiological process involving multiple genetic alterations (4,5). Matrix metalloproteinases (MMPs), particularly MMP-2 and MMP-9, play an important role in the invasion process of numerous malignant tumors by degrading the basement membrane and extracellular matrix (ECM) (6-8). Previous studies have suggested that MMP-2 and MMP-9 are associated with invasion in lung cancer $(4,5)$. In addition, numerous studies have demonstrated that MMPs may be regulated by tissue inhibitor of metalloproteinase (TIMP) $(6,9)$. Disturbing the balance of MMPs and TIMPs may affect the remodeling, formation and degradation of matrix protein and induce invasion of cancer cells.

The phosphoinositide 3-kinase (PI3K)/AKT signaling pathway performs a key role in the control of cell differentiation and proliferation. Previously, activation of the PI3K/AKT signaling pathway was suggested to be associated with the invasion of numerous tumor types, including prostate, ovarian, colon and breast cancers (10-13). In lung cancer, the PI3K/AKT signaling pathway is also considered as a crucial activator of intracellular signaling cascades in invasion progression and is useful as a therapeutic target for anticancer drug development. 
Allicin, the main active principle associated with Allium sativum chemistry, possesses therapeutic potential, with antioxidant, anti-inflammatory and antitumor activities. Previous studies demonstrated that allicin may induce tumor cell apoptosis, inhibit the tumor cell cycle and regulate angiogenesis (14-17). Numerous mechanisms are involved in the biological activities of allicin, including unfolded protein response, p53-mediated autophagy and p38 mitogen-activated protein kinase/caspase-3 signaling (14-18). However, to the best of our knowledge, the effects and mechanisms of allicin on lung adenocarcinoma remain undefined. In addition, the role of allicin in inhibiting invasion has not been reported. In the present study, it was revealed that allicin may suppress migration and invasion of lung adenocarcinoma cells by altering TIMP/MMP balance via reduction of the activity of the PI3K/AKT signaling pathway in vitro.

\section{Materials and methods}

Reagents. Lipofectamine ${ }^{\circledR} 2000$ reagents and fetal bovine serum (FBS) were purchased from Invitrogen (Thermo Fisher Scientific, Inc., Waltham, MA, USA). Allicin and specific activator of PI3K (insulin-like growth factor-1; IGF-1) were purchased from Santa Cruz Biotechnology, Inc. (Dallas, TX, USA). Specific inhibitor of PI3K (LY294002) was purchased from Beyotime Institute of Biotechnology (Shanghai, China). MTT was purchased from Beyotime Institute of Biotechnology. Anti-phospho-AKT ${ }^{\text {Ser473 }}$ (anti-p-AKT ${ }^{\text {S473 }}$ ) and anti-AKT were purchased from Cell Signaling Technology, Inc. (Danvers, MA, USA). Anti-MMP-2, anti-MMP-9, anti-TIMP-1, anti-TIMP-2 and anti- $\beta$-actin were purchased from Santa Cruz Biotechnology, Inc. Horseradish peroxidase (HRP)-conjugated goat anti-rabbit (cat no. ZDR-5307; dilution, 1:2,000) and anti-mouse immunoglobulin (cat no. ZDR-5307; dilution, 1:2,000) were obtained from Beijing Zhongshan Golden Bridge Biotechnology Co., Ltd. (Beijing, China).

Cell lines and cell culture. The two lung adenocarcinoma cell lines A549 and H1299 were purchased from the Cell Bank of Type Culture Collection of Chinese Academy of Sciences (Shanghai, China). The cells were cultured in Dulbecco's modified Eagle's medium with 10\% FBS, $100 \mathrm{U} / \mathrm{ml}$ penicillin and $100 \mathrm{mg} / \mathrm{ml}$ streptomycin at $37^{\circ} \mathrm{C}$ in a humidified atmosphere with $5 \% \mathrm{CO}_{2}$.

Cell viability assay. The effects of allicin on cell viability were determined by an MTT assay. MTT was purchased from Beyotime Institute of Biotechnology. Cells $\left(10^{4}\right.$ cells per well $)$ were seeded onto 96 -well plates, incubated overnight at $37^{\circ} \mathrm{C}$ and then incubated in various concentrations of allicin $(0,1.0$, 2.5, 5.0, 7.5, 10.0, 15.0 and $20.0 \mu \mathrm{M})$ for $24 \mathrm{~h}$. The medium was then removed and the MTT solution $(0.5 \mathrm{mg} / \mathrm{ml})$ was added to the cell culture. Following incubation for $4 \mathrm{~h}$ at $37^{\circ} \mathrm{C}$, the reaction was stopped by adding dimethyl sulfoxide $(0.5 \mathrm{mg} / \mathrm{ml})$. At the end, absorbance was measured spectrophotometrically at 570 nm (Bio-Tek ELX800UV; Omega Bio-Tek Inc., Norcross, GA, USA).

Cell adhesion assay. The 96-well plates were prepared for coating with $5 \mathrm{mg} / \mathrm{ml}$ fibronectin (Sigma-Aldrich; EMD
Millipore, Billerica, MA, USA) and blocking with $1 \%$ bovine serum albumin (BSA) for $4 \mathrm{~h}$. A549 and H1299 cells were incubated for $48 \mathrm{~h}$ with various concentrations of allicin $(0,5.0,7.5$ and $10.0 \mu \mathrm{M})$ at $37^{\circ} \mathrm{C}$. Cells $(20,000$ cells/well $)$ were then allowed to attach to fibronectin coated plates for $1 \mathrm{~h}$ at $37^{\circ} \mathrm{C}$. The unattached cells were washed away with PBS. Attached cells were quantified by MTT assay.

Transwell migration and invasion assays. Cell invasion experiments were assayed using 6.5-mm Transwell chambers (8- $\mu \mathrm{m}$ pore size; Corning-Costar Inc., Corning, NY, USA). The filters were precoated with 1-2 mg/ml Matrigel (reconstituted basement membrane; BD Biosciences, Franklin Lakes, CA, USA). Cells were pretreated with $0,5.0,7.5$ and $10.0 \mu \mathrm{M}$ allicin or IGF-1 (50 ng/ml) or LY294002 (25 $\mu \mathrm{M})$. Surviving cells in $100 \mu \mathrm{l}$ of serum-free medium were seeded in the upper chamber. Medium supplemented with 10\% FBS was added to the lower chamber as the chemoattractant. Following $24 \mathrm{~h}$ of incubation at $37^{\circ} \mathrm{C}$, the cells on the upper side were wiped with a cotton bud. The cells that had migrated into the lower compartment were fixed with methanol, stained with hematoxylin and eosin (Beyotime Institute of Biotechnology) and counted in 5 random fields of the insert under a light microscope (magnifcation, x200). A migration assay was performed as described for the invasion assay, but with a shorter incubation period (12 h) and no Matrigel coating.

Western blot analysis. The A549 and H1299 cells were extracted with radioimmunoprecipitation assay buffer [1 $\mathrm{mg} / \mathrm{ml}$ phenylmethylsulfonyl fluoride, $1 \mathrm{Mm}$ aprotinin, $1 \mathrm{mg} / \mathrm{ml}$ leupeptin, $1 \mathrm{mM}$ EDTA, $150 \mathrm{mM} \mathrm{NaCl}, 0.25 \%$ Na-deoxycholate, $1 \mathrm{mg} / \mathrm{ml}$ pepstatin and $50 \mathrm{mM}$ Tris- $\mathrm{HCl}$ ( $\mathrm{pH}$ 7.4)] following treatment with various concentrations $(0$, 5.0, 7.5 and $10.0 \mu \mathrm{M}$ ) of allicin, IGF-1 or LY294002. Total proteins were quantified using the bicinchoninic acid method. Equal amounts of protein were separated on SDS-PAGE gels and electrophoretically transferred to polyvinylidene fluoride membranes. Subsequent to blocking in 5\% BSA for $1 \mathrm{~h}$ at room temperature, membranes were incubated overnight at $4^{\circ} \mathrm{C}$ with antibodies against MMP-2 (dilution, 1:500; cat no. sc-13594), MMP-9 (dilution, 1:500; cat no. sc-12759), TIMP-1 (dilution, 1:500; cat no. sc-6832), TIMP-2 (dilution, 1:500; cat no. sc-365671), p-AKT ${ }^{\mathrm{S} 473}$ (dilution, 1:1,000; cat no. sc-33437), AKT (dilution, 1:1,000; cat no. sc-24500) or $\beta$-actin (dilution, 1:1,000; cat no. sc-10731). The membranes were then incubated with the appropriate HRP-conjugated goat anti-rabbit (cat no. ZDR-5307; dilution, 1:2,000) and anti-mouse immunoglobulin (cat no. ZDR-5307; dilution, $1: 2,000)$ for $1 \mathrm{~h}$ at room temperature. The bands were visualized by enhanced chemiluminescence (Thermo Fisher Scientific, Inc.). The densitometry analysis was performed using Quality One analysis software (version 6.0; Bio-Rad Laboratories, Inc., Hercules, CA, USA).

Reverse transcription-quantitative polymerase chain reaction (RT-qPCR). Total RNA was extracted from A549 and H1299 cells using TRIzol (Invitrogen; Thermo Fisher Scientific, Inc.), subsequent to treatment with various concentrations of allicin $(0,5.0,7.5$ and $10.0 \mu \mathrm{M})$. cDNA was synthesized 
and RT-qPCR was performed in accordance with previously described protocols (19). The primers for human MMP-2 (forward, 5'-GGTTGTCTGAAGTCACTGCACAGT-3' and reverse, 5'-CTCGGTAGGGACATGCTAAGTAGAG-3'), MMP-9 (forward, 5'-GCTGGGCTTAGATCATTCCTCA-3' and reverse, 5'-CTGGCGACGCAAAAGAAGA-3'), TIMP-1 (forward, 5'-GAGAACCCACCATGGCCC-3' and reverse, 5'-TATCAGCCACAGCAACAACAGG-3'), TIMP-2 (forward, 5'-CCACCCAGAAGAAGAGCCTG-3' and reverse, 5'-CAG CGCGTGATCTTGCAC-3') and GAPDH (forward, 5'-CCT CCCGCTTCGCTCTCT-3' and reverse, 5'-CTGGCGACG CAAAAGAAGA-3') were used for RT-qPCR. The average expression level of genes was normalized to the reference gene GAPDH. Data analysis was performed using the $2^{-\Delta \Delta \mathrm{Cq}}$ method (19).

Statistical analysis. Each experiment was repeated at least three times. Data are presented as the mean \pm standard deviation. SPSS version 16.0 statistical software (SPSS, Inc., Chicago, IL, USA) and Student's t-test were used for statistical analysis. $\mathrm{P}<0.05$ was considered to indicate a statistically significant difference.

\section{Results}

Allicin inhibits proliferation of lung adenocarcinoma cells. To determine the antitumor effect of allicin against lung adenocarcinoma cells, the ability of allicin to inhibit proliferation in lung adenocarcinoma A549 and H1299 cells was first examined by the cell viability assay. As shown in Fig. 1, cell proliferation was significantly reduced in A549 and H1299 cells following treatment with 15.0 and $20.0 \mu \mathrm{M}$ allicin $(\mathrm{P}<0.000)$. However, no significant reduction in proliferation was observed when lung adenocarcinoma cells were treated with allicin at concentrations below $15.0 \mu \mathrm{M}$. Therefore, a concentration range of allicin $<15.0 \mu \mathrm{M}$ was selected for all subsequent experiments in order to exclude the effect of cellular cytotoxicity on invasion.

Allicin inhibits adhesion, migration and invasion of lung adenocarcinoma cells. Cell migration and invasion are critical events in the development of lung adenocarcinoma. The present study first examined the cell adhesion ability following incubation of A549 and H1299 cells with allicin. It was observed that allicin treatment decreased tumor cell adhesion to fibronectin in a concentration-dependent manner (Fig. 2A and B). The effect of allicin on migration and invasion was then examined in A549 and H1299 cells that were exposed to various concentrations of allicin for $12 \mathrm{~h}$ (cell migration) and $24 \mathrm{~h}$ (cell invasion). The number of migratory or invasive lung adenocarcinoma cells decreased in a dose-dependent manner (Fig. 2C and D). The aforementioned data demonstrated that allicin inhibited adhesion, migration and invasion of lung adenocarcinoma cells.

Allicin alters TIMP/MMP balance in lung adenocarcinoma cells. It has been demonstrated that MMPs, particularly MMP-2 and MMP-9, are involved in the invasion of lung cancer. Therefore, the present study then investigated whether allicin regulates the expression of MMP-2 and MMP-9. Allicin was revealed to dose-dependently inhibit MMP-2 and MMP-9 mRNA and protein levels in $\mathrm{H} 1299$ cells (Fig. 3). Previous studies indicated that the expression of MMPs was regulated by their endogenous tissue inhibitors (TIMPs) (7-9). Thus, the expression levels of TIMP-1 and TIMP-2 in H1299 cells were examined by RT-qPCR and western blot analysis following treatment with $0,5.0,7.5$ and $10.0 \mu \mathrm{M}$ of allicin for $48 \mathrm{~h}$. It was identified that allicin upregulated the RNA and protein levels of TIMP-1 and TIMP-2 in H1299 cells in a concentration-dependent manner (Fig. 3). These results indicated that allicin regulates TIMP/MMP balance and stimulates H1299 cell invasion.

The PI3K/AKT signaling pathway is associated with the anti-invasive mechanism of allicin. Studies have shown that activation of PI3K/AKT signaling plays a vital role in the invasion process of lung cancer $(12,20)$. Thus, the effect of allicin on the PI3K/AKT signaling pathway in H1299 cells was investigated. As shown in Fig. 4, allicin significantly suppressed the phosphorylation of AKT in a concentration-dependent manner $(\mathrm{P}<0.05)$. However, the total protein expression of AKT was not altered by allicin. To confirm whether the inhibitory effect of allicin on cell invasion and TIMP/MMP balance was associated with inhibition of the PI3K/AKT signaling pathway, H1299 cells were pretreated with or without PI3K inhibitor (LY294002, $25 \mu \mathrm{M}$ ) for $1 \mathrm{~h}$, and then exposed to allicin $(0$ or $7.5 \mu \mathrm{M})$ for $48 \mathrm{~h}$. It was identified that treatment with LY294002 and allicin significantly inhibited cell invasion $(\mathrm{P}<0.015$; Fig. 5A), decreased MMP-2 $(\mathrm{P}<0.002)$ and MMP-9 $(\mathrm{P}<0.000)$ protein expression and increased TIMP-1 $(\mathrm{P}<0.000)$ and TIMP-2 $(\mathrm{P}<0.000)$ protein expression (Fig. 5B and $\mathrm{C}$ ) compared with the allicin treated group. Furthermore, H1299 cells were pretreated with PI3K activator (IGF-1; 0 or $50 \mathrm{ng} / \mathrm{ml}$ ) for $1 \mathrm{~h}$ and then exposed to various concentrations of allicin $(0$ or $7.5 \mu \mathrm{M})$ for $48 \mathrm{~h}$. It was revealed that the effects of allicin on cell invasion and protein expression of MMP-2 $(\mathrm{P}<0.001)$, MMP-9 $(\mathrm{P}<0.001)$, TIMP-1 $(\mathrm{P}<0.000)$ and TIMP-2 $(\mathrm{P}<0.000)$ were significantly reversed by IGF-1 (Fig. 5D-F). These results revealed that allicin inhibited the invasion of lung adenocarcinoma cells by altering TIMP/MMP balance via regulation of $\mathrm{PI} 3 \mathrm{~K} / \mathrm{AKT}$ signaling.

\section{Discussion}

Abnormal invasion of cancer cells is considered to be the crucial biological feature of cancer. The presence of invasion is the major reason of recurrence and mortality in patients with lung cancer $(1,20)$. In the present study, allicin was found to inhibit lung adenocarcinoma cell adhesion, migration and invasion. It also provided evidence that the mechanism underlying the aforementioned effects was associated with altering TIMP/MMP balance, which was regulated by the PI3K/AKT signaling pathway. The present study shed light on the investigation of allicin in lung adenocarcinoma invasion.

Increasing studies have demonstrated that allicin exhibits a cytotoxic effect in several human cancer cells, including glioma U87, liver cancer G2 and gastric cancer MGC803 cells (15-17). These high specificities make allicin a promising anticancer agent for lung adenocarcinoma. In the present study, it was identified that treatment with allicin was able to 
A

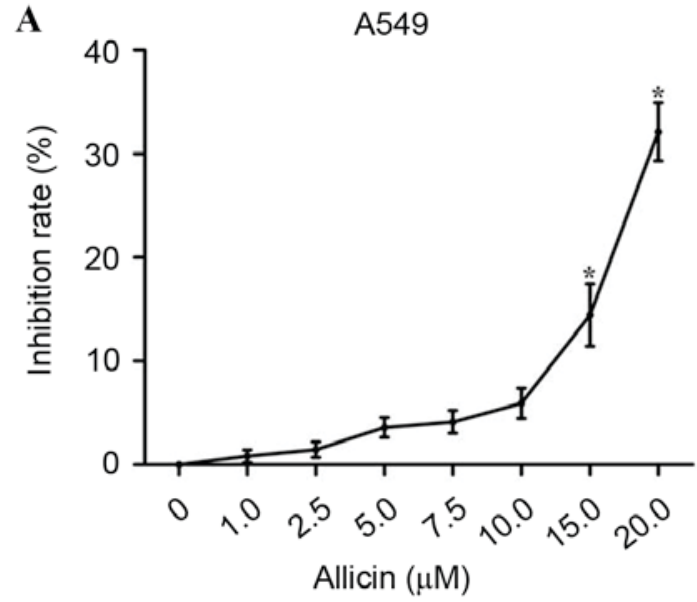

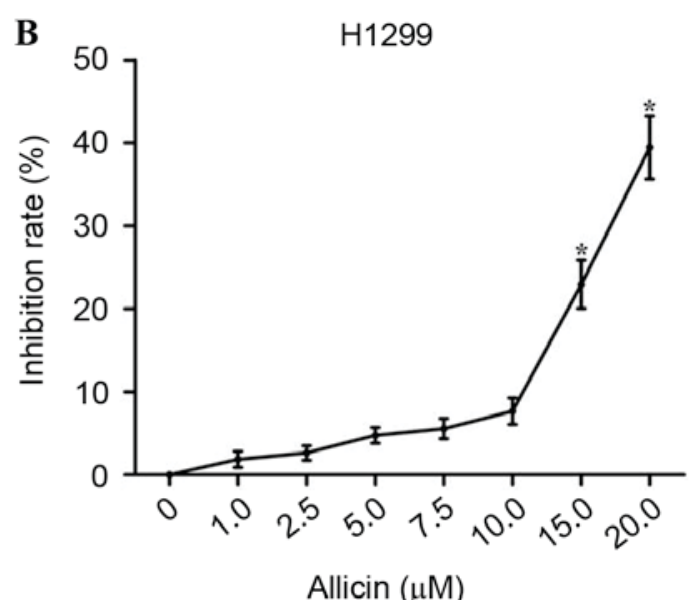

Allicin $(\mu \mathrm{M})$

Figure 1. Effects of allicin on the proliferation of lung adenocarcinoma cells. (A) A549 and (B) H1299 cells were treated with different concentrations of allicin for $24 \mathrm{~h}$. Cell viability was determined by the MTT method. Data are expressed as the mean \pm standard deviation of three independent experiments. ${ }^{*} \mathrm{P}<0.05$ compared with the $0 \mu \mathrm{M}$ arctigenin group.

A

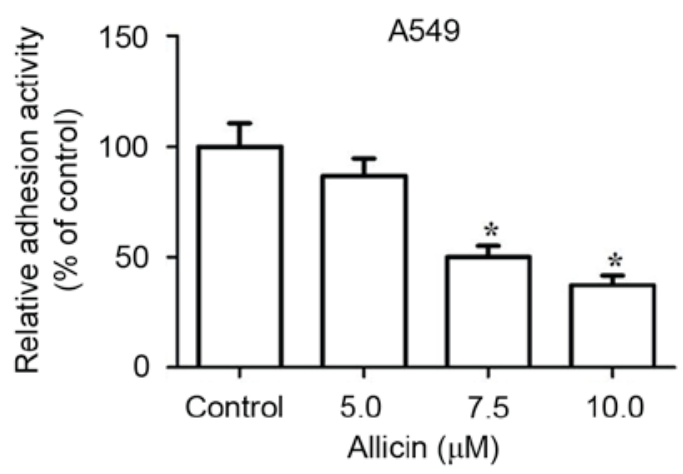

C

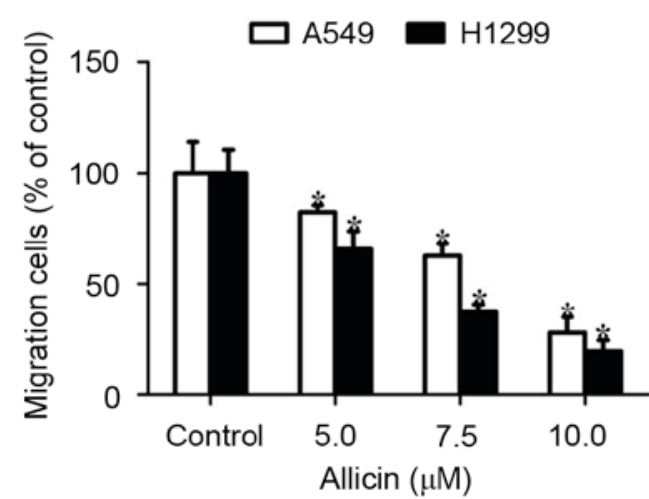

B

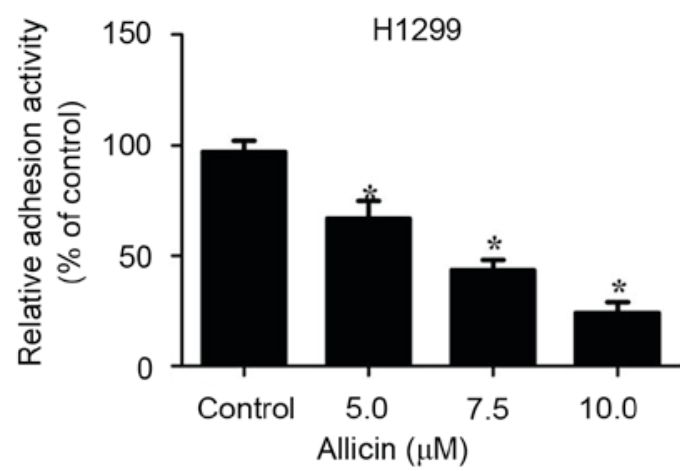

D

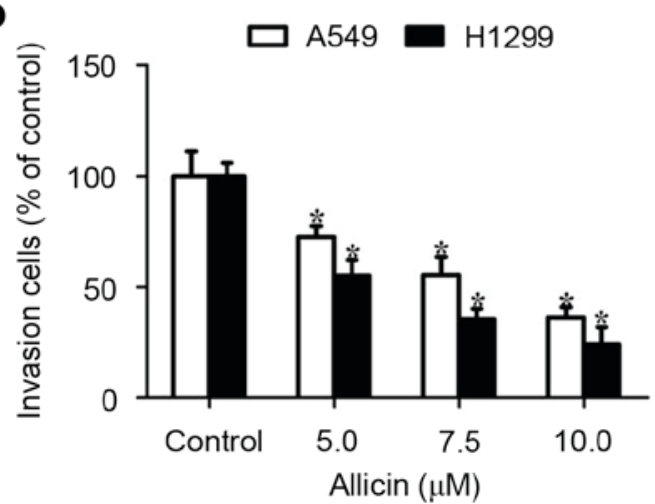

Figure 2. Effect of allicin on the adhesion, migration and invasion of lung adenocarcinoma cells. (A) A549 and (B) H1299 cells were incubated for $24 \mathrm{~h}$ with various concentrations of allicin. Cells were seeded onto 96-well plates coated with fibronectin. After $1 \mathrm{~h}$, the adhered cells were analyzed by MTT assay. The adhesion rate was expressed as a percentage of the control $(0 \mu \mathrm{M})$. (C) A549 and H1299 cells were seeded in the upper wells without coating of Matrigel, and treated with various concentrations of allicin. After $12 \mathrm{~h}$, cells on the bottom side of the filter were fixed, stained and counted. The migration rate was expressed as a percentage of the control $(0 \mu \mathrm{M})$. (D) A549 and H1299 cells were seeded in the upper wells with coating of Matrigel, and treated with various concentrations of allicin. After $24 \mathrm{~h}$, cells on the bottom side of the filter were fixed, stained and counted. The invasion rate was expressed as a percentage of the control $(0 \mu \mathrm{M})$. Data are expressed as the mean \pm standard deviation of three independent experiments. ${ }^{*} \mathrm{P}<0.05$ compared with controls.

suppress cell viability, indicating that allicin also possesses cytotoxicity against lung adenocarcinoma. To the best of our knowledge, there are only a small number of studies on the association between allicin and the invasion of cancer cells. Thus, the role of allicin in the invasion of lung adenocarcinoma cells was also analyzed. It was revealed that allicin is associated with decreased adhesion, migration and invasion of lung adenocarcinoma cells. This indicated that allicin may suppress invasiveness in lung adenocarcinoma.

MMPs, particularly MMP-2 and MMP-9, control cell-cell and cell-matrix interactions. Normally, TIMPs specifically combine with MMPs and keep their activity in a dynamic 
A

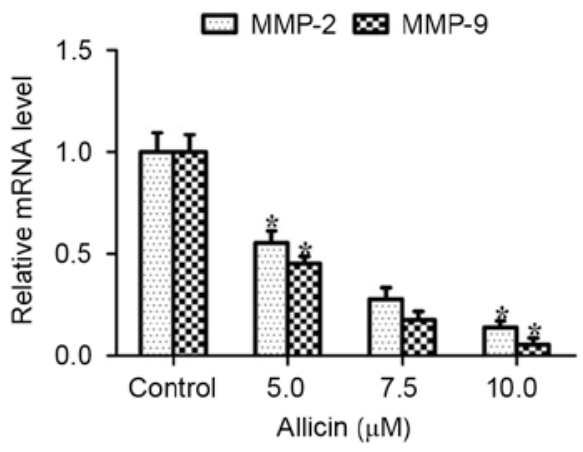

C

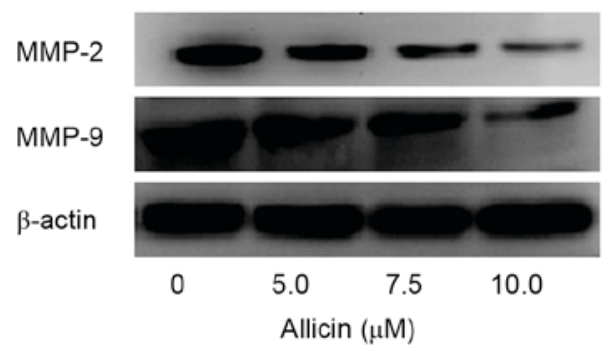

E

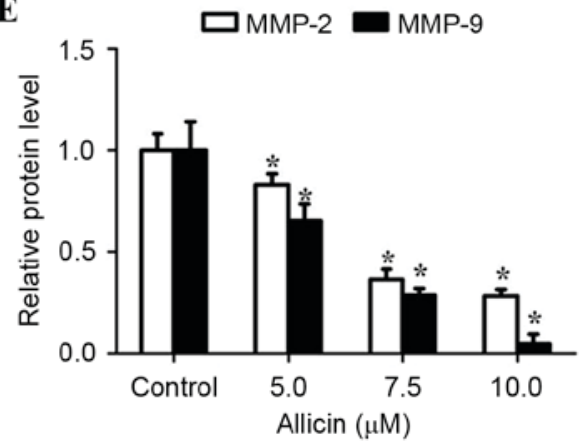

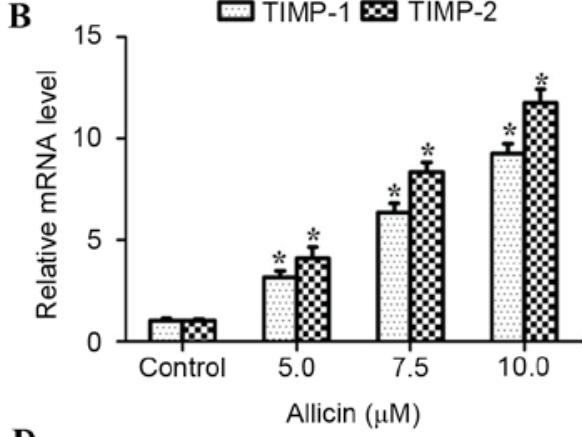

D

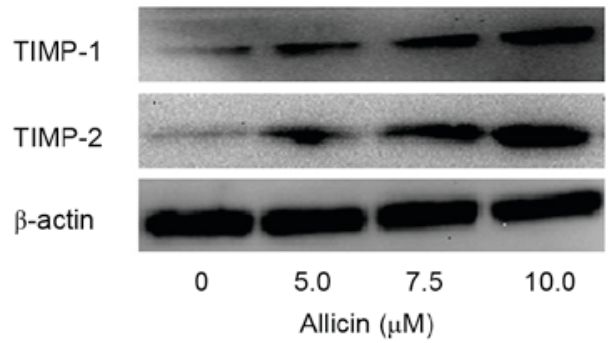

F

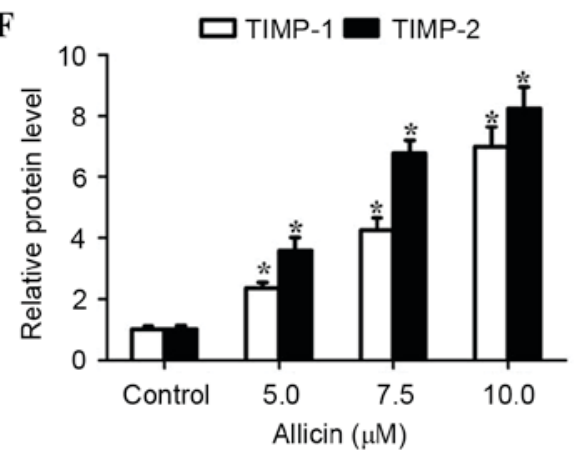

Figure 3. Allicin alters TIMP/MMP balance in H1299 cells. H1299 cells were treated with 0, 5.0, 7.5 and $10.0 \mu \mathrm{M}$ allicin for $48 \mathrm{~h}$ and the mRNA levels of (A) MMP-2 and MMP-9, and (B) TIMP-1 and TIMP-2 were assayed by reverse transcription-quantitative polymerase chain reaction and normalized to GAPDH. H1299 cells were incubated with various concentrations of allicin (0, 5.0, 7.5 and $10.0 \mu \mathrm{M})$ for $48 \mathrm{~h}$. The protein levels of (C) MMP-2, MMP-9, (D) TIMP-1 and TIMP-2 in cell lysates were analyzed by western blot analysis and band intensity was quantified by densitometry and normalized to $\beta$-actin for (E) MMP-2 and MMP-9 and (F) TIMP-1 and TIMP-2. Values are presented as the mean \pm standard deviation of three independent experiments. "P<0.05, compared with control $(0 \mu \mathrm{M})$. MMP, matrix metalloproteinase; TIMP, tissue inhibitor of metalloproteinase.

A

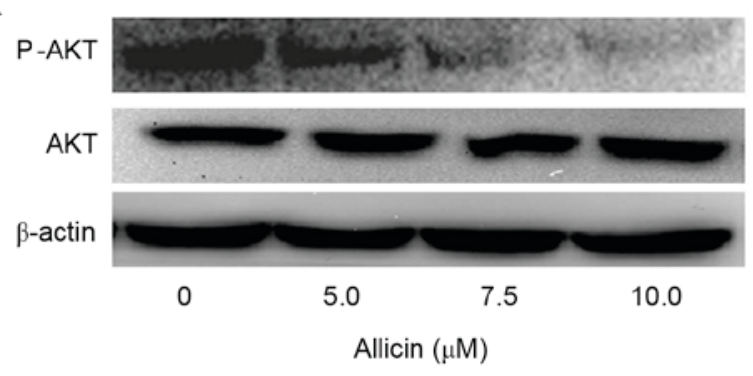

B

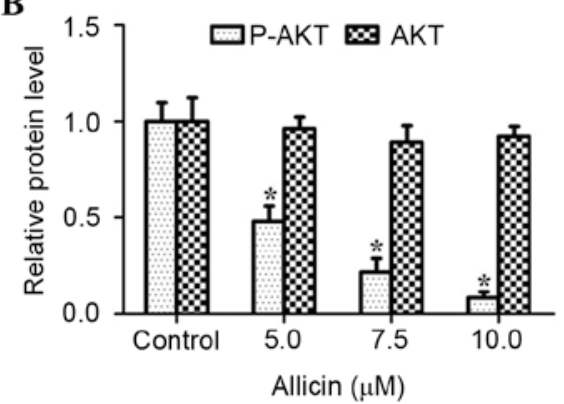

Figure 4. Effects of allicin on the PI3K/AKT signaling pathway. (A) H1299 cells were incubated with various concentrations of allicin $(0,5.0,7.5$ and $10 \mu \mathrm{M})$ for $48 \mathrm{~h}$. The protein levels of P-AKT and AKT in cell lysates were analyzed by western blot analysis. (B) The band intensity was quantified by densitometry and normalized to $\beta$-actin. Values are expressed as the mean \pm standard deviation of three independent experiments. ${ }^{*} \mathrm{P}<0.05$, compared with the control $(0 \mu \mathrm{M})$. P-AKT, phospho-AKT.

balance. However, once this balance is broken, invasion and metastasis of cancer cells is induced (6-9). The imbalance between TIMPs and MMPs has been recognized as the main mechanism for promoting the invasive processes of lung cancer.
Hu et al reported that hypoxia may affect the invasiveness of lung cancer cells by regulating MMP-9 and TIMP-2 expression (21). Ylisirnio et al demonstrated that serum MMP-2, MMP-9, TIMP-1 and TIMP-2 were associated with the 

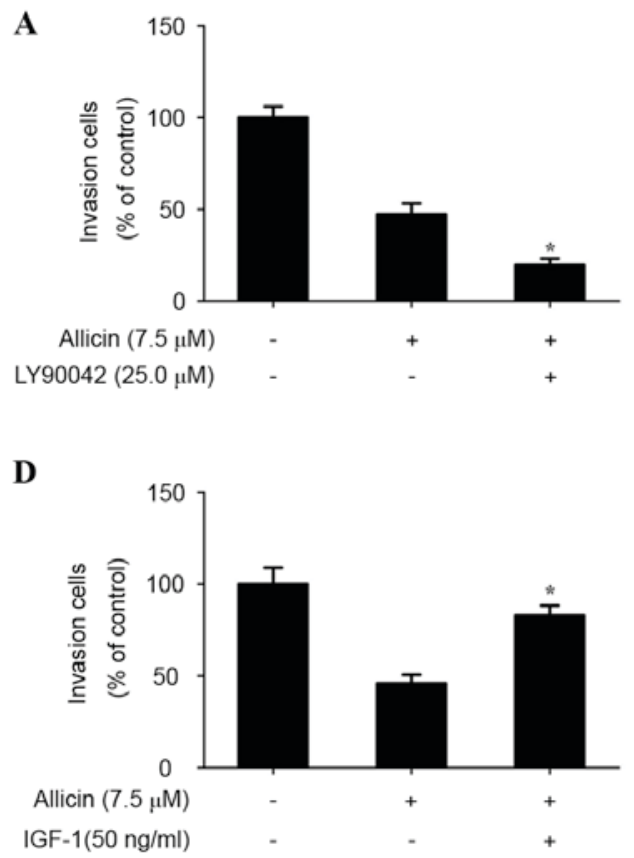

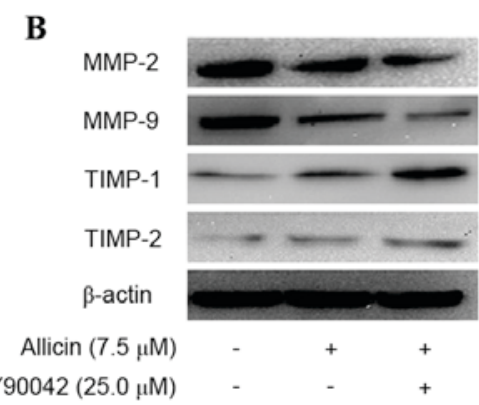

E

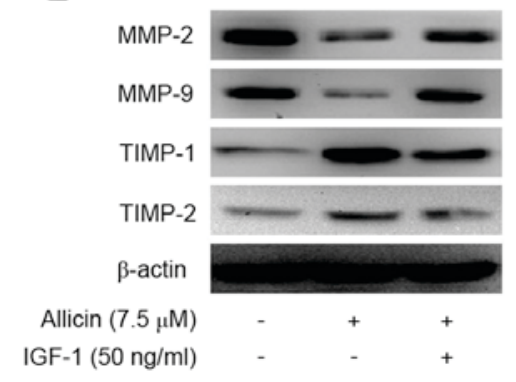

C

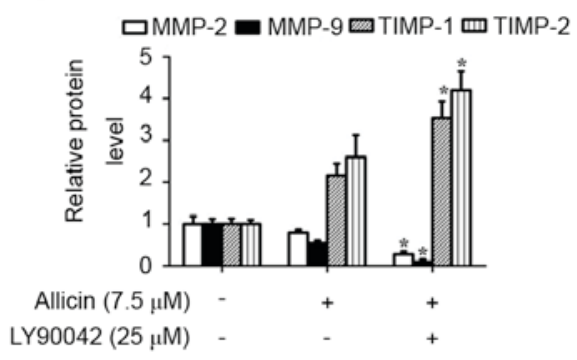

$\mathbf{F}$

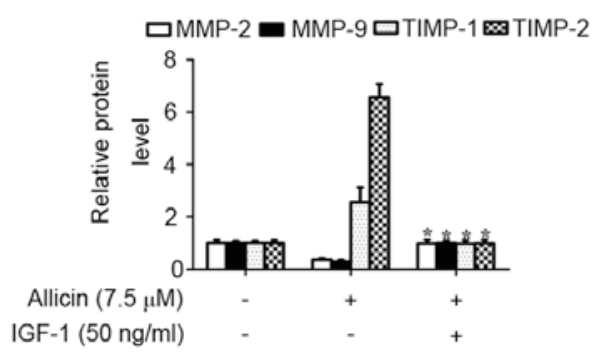

Figure 5. Involvement of the PI3K/AKT pathway in allicin-induced TIMP/MMP imbalance and cell invasion. (A) Cells were pretreated with LY294002 $(25 \mu \mathrm{M})$ for $1 \mathrm{~h}$ and then incubated in the presence or absence of allicin $(7.5 \mu \mathrm{M})$ for $48 \mathrm{~h}$. Cellular invasiveness was measured using the Transwell invasion assay. The invasion rate was expressed as a percentage of control. (B) The protein levels of MMP-2, MMP-9, TIMP-1 and TIMP-2 were analyzed in treated H1299 cells by western blotting. (C) Values are expressed as the mean \pm SD of three independent experiments. "P<0.05, compared with the allicin treated group. (D) Cells were pretreated with IGF-1 $(50 \mathrm{ng} / \mathrm{ml})$ for $1 \mathrm{~h}$ and then incubated in the presence or absence of allicin $(7.5 \mu \mathrm{M})$ for $48 \mathrm{~h}$. Cellular invasiveness was measured using the Transwell invasion assay. The invasion rate was expressed as a percentage of the control. (E) The protein levels of MMP-2, MMP-9, TIMP-1 and TIMP-2 were analyzed in treated H1299 cells by western blot analysis. (F) Band intensity was quantified by densitometry and normalized to $\beta$-actin. Values are expressed as the mean $\pm \mathrm{SD}$ of three independent experiments. ${ }^{*} \mathrm{P}<0.05$ compared with the allicin treated group. MMP, matrix metalloproteinase; TIMP, tissue inhibitor of metalloproteinase; IGF-1, insulin-like growth factor-1; SD, standard deviation; PI3K, phosphoinositide 3-kinase.

clinical outcome of patients with lung cancer and may serve as prognostic markers (22). To explore the possible mechanism of allicin in the inhibition of lung adenocarcinoma invasion, the effects of allicin on the expression level of MMP-2, MMP-9, TIMP-1 and TIMP-2 were investigated. It was revealed that allicin dose-dependently downregulated mRNA and protein levels of MMP-2 and MMP-9 and then enhanced mRNA and protein levels of TIMP-1 and TIMP-2 in a dose-dependent manner. These data indicated that allicin may cause inhibition of lung adenocarcinoma invasion by inducing an imbalance of expression between MMPs (MMP-2 and MMP-9) and TIMPs (TIMP-1 and TIMP-2).

Previous studies established that the PI3K/AKT pathway is activated in numerous tumors, including breast cancers, pituitary adenoma and prostate cancer $(10,13)$. An increasing number of studies have shown that the PI3K/AKT signaling pathway may modulate the expression of MMPs, as well as TIMPs, to promote the degradation of ECM proteins, and this mechanism was essential for invasion of human tumors, including lung cancer $(13,23)$. To the best of our knowledge, allicin has been confirmed to inhibit the PI3K/AKT signaling pathway in the HepG2 cell line; however, it remains unknown whether such an effect also exists in lung adenocarcinoma (17). Therefore, the effect of allicin on the PI3K/AKT signaling pathway was investigated in H1299 cells. The results demonstrated that allicin may decrease the phosphorylation of AKT in H1299 cells, whereas no significant changes were observed in the total protein expression of AKT. In addition, allicin combined with LY294002 (an inhibitor of PI3K) significantly reduced H1299 cell invasion $(\mathrm{P}<0.05)$ and was accompanied by upregulation of TIMP-1 and TIMP-2 and downregulation of MMP-2 and MMP-9. Whereas, in H1299 cells, the PI3K/AKT signaling activator (IGF-1) reversed the effect produced by allicin on invasion, as well as the protein expression of MMP-2, MMP-9, TIMP-1 and TIMP-2. These findings indicated that the regulation of cell invasion and TIMP-1, TIMP-2, MMP-2 and MMP-9 expression by allicin occurred via the suppression of the PI3K/AKT signaling pathway.

In summary, to the best of our knowledge, the present data demonstrated for the first time that allicin inhibits the invasion of lung adenocarcinoma cells by altering TIMP/MMP balance, via reducing the activity of the PI3K/AKT signaling pathway. Allicin may be recognized as an anti-invasive agent for lung adenocarcinoma treatment.

\section{References}

1. Zhang XD, Li W, Zhang N, Hou YL, Niu ZQ, Zhong YJ, Zhang YP and Yang SY: Identification of adipophilin as a potential diagnostic tumor marker for lung adenocarcinoma. Int J Clin Exp Med 7: 1190-1196, 2014.

2. Pannu BS and Iyer VN: Lung adenocarcinoma presenting with isolated 'chronic cough' of 3 years duration-a cautionary tale. Respir Med Case Rep 16: 157-159, 2015.

3. Sasada S, Miyata Y, Mimae T, Tsutani Y, Mimura T and Okada M: Application of PET/CT to adjuvant chemotherapy for early lung adenocarcinoma. J Cardiothorac Surg 10 (Suppl 1): A198, 2015.

4. Wang L, Zhan W, Xie S, Hu J, Shi Q, Zhou X, Wu Y, Wang S, Fei Z and Yu R: Over-expression of Rap2a inhibits glioma migration and invasion by down-regulating p-AKT. Cell Biol Int 38: 326-334, 2014. 
5. Huang LC and Hueng DY: CD97 and glioma invasion. J Neurosurg 120: 579-580, 2014.

6. Dung TD, Feng CC, Kuo WW, Pai P, Chung LC, Chang SH, Hsu HH, Tsai FJ, Lin YM and Huang CY: Suppression of plasminogen activators and the MMP-2/-9 pathway by a Zanthoxylum avicennae extract to inhibit the HA22T human hepatocellular carcinoma cell migration and invasion effects in vitro and in vivo via phosphatase $2 \mathrm{~A}$ activation. Biosci Biotechnol Biochem 77: 1814-1821, 2013

7. Gutschalk CM, Yanamandra AK, Linde N, Meides A, Depner S and Mueller MM: GM-CSF enhances tumor invasion by elevated MMP-2, -9, and -26 expression. Cancer Med 2: 117-129, 2013.

8. Zhang C, Li Y, Qian ZJ, Lee SH, Li YX and Kim SK: Dieckol from Ecklonia cava Regulates Invasion of Human Fibrosarcoma Cells and Modulates MMP-2 and MMP-9 Expression via NF- $\kappa$ B Pathway. Evid Based Complement Alternat Med 2011: 140462 , 2011.

9. Krengel S, Alexander M, Brinckmann J and Tronnier M: MMP-2, TIMP-2 and MT1-MMP are differentially expressed in lesional skin of melanocytic nevi and their expression is modulated by UVB-light. J Cutan Pathol 29: 390-396, 2002.

10. Lee YR, Park J, Yu HN, Kim JS, Youn HJ and Jung SH: Up-regulation of PI3K/Akt signaling by 17 beta-estradiol through activation of estrogen receptor-alpha, but not estrogen receptor-beta, and stimulates cell growth in breast cancer cells. Biochem Biophys Res Commun 336: 1221-1226, 2005.

11. Thamilselvan V, Craig DH and Basson MD: FAK association with multiple signal proteins mediates pressure-induced colon cancer cell adhesion via a Src-dependent PI3K/Akt pathway. FASEB J 21: 1730-1741, 2007.

12. Meng Q, Xia C, Fang J, Rojanasakul Y and Jiang BH: Role of $\mathrm{PI} 3 \mathrm{~K}$ and AKT specific isoforms in ovarian cancer cell migration, invasion and proliferation through the p70S6K1 pathway. Cell Signal 18: 2262-2271, 2006.

13. Ni J, Cozzi P, Hao J, Beretov J, Chang L, Duan W, Shigdar S, Delprado W, Graham P, Bucci J, et al: Epithelial cell adhesion molecule (EpCAM) is associated with prostate cancer metastasis and chemo/radioresistance via the PI3K/Akt/mTOR signaling pathway. Int J Biochem Cell Biol 45: 2736-2748, 2013.
14. Luo R, Fang D, Hang H and Tang Z: Recent progress of allicin on cell growth inhibition and Apoptosis in gastric cancer cells. Anticancer Agents Med Chem 16: 802-809, 2016.

15. Zhang X, Zhu Y, Duan W, Feng C and He X: Allicin induces apoptosis of the MGC-803 human gastric carcinoma cell line through the p38 mitogen-activated protein kinase/caspase-3 signaling pathway. Mol Med Rep 11: 2755-2760, 2015.

16. Chu YL, Ho CT, Chung JG, Rajasekaran R and Sheen LY: Allicin induces p53-mediated autophagy in Hep G2 human liver cancer cells. J Agric Food Chem 60: 8363-8371, 2012.

17. Cha JH, Choi YJ, Cha SH, Choi $\mathrm{CH}$ and Cho WH: Allicin inhibits cell growth and induces apoptosis in U87MG human glioblastoma cells through an ERK-dependent pathway. Oncol Rep 28: 41-48, 2012.

18. Bat-Chen W, Golan T, Peri I, Ludmer Z and Schwartz B: Allicin purified from fresh garlic cloves induces apoptosis in colon cancer cells via Nrf2. Nutr Cancer 62: 947-957, 2010.

19. Zhou QX, Jiang XM, Wang ZD, Li CL and Cui YF: Enhanced expression of suppresser of cytokine signaling 3 inhibits the IL-6-induced epithelial-to-mesenchymal transition and cholangiocarcinoma cell metastasis. Med Oncol 32: 105, 2015.

20. Zhang P, Yu S, Li H, Liu C, Li J, Lin W, Gao A, Wang L, Gao W and Sun Y: ILT4 drives B7-H3 expression via PI3K/AKT/mTOR signalling and ILT4/B7-H3 co-expression correlates with poor prognosis in non-small cell lung cancer. FEBS Lett 589: 2248-2256, 2015.

21. Hu Z, Huang J, Li Q, Yang J, Zhong L and Zeng Q: Effect of hypoxia on infiltration and migration of lung cancer cells and expression of MMP-2 and TIMP-2. Zhongguo Fei Ai Za Zhi 8: 270-273, 2005 (In Chinese).

22. Ylisirnio S, Höyhtyä M and Turpeenniemi-Hujanen T: Serum matrix metalloproteinases $-2,-9$ and tissue inhibitors of metalloproteinases $-1,-2$ in lung cancer-TIMP-1 as a prognostic marker. Anticancer Res 20: 1311-1316, 2000.

23. Chen YY, Liu FC, Chou PY, Chien YC, Chang WS, Huang GJ, Wu CH and Sheu MJ: Ethanol extracts of fruiting bodies of Antrodia cinnamomea suppress CL1-5 human lung adenocarcinoma cells migration by inhibiting matrix metalloproteinase-2/9 through ERK, JNK, p38, and PI3K/Akt signaling pathways. Evid Based Complement Alternat Med 2012: 378415, 2012. 